\title{
DIRECTORY OF IUGS BODIES
}

\section{COMMISSIONS}

COMMISSION ON COMPARATIVE PLANETOLOGY (CCP) Secretary General: Dr. R.A.F. Grieve, Geological Survey of Canada, 1 Observatory Crescent, Ottawa, Canada K1A 0Y3.

COMMISSION ON GEOLOGICAL DOCUMENTATION (COGEODOC) Secretary-Treasurer: Dr. J. Gravesteijn, Dépt. Doc/BRGM, BP 6009, F-45018 Orléans-Cedex, France.

COMMISSION ON GEOLOGY TEACHING (CGT)

Chair: vacant.

COMMISSION ON GLOBAL SEDIMENTARY GEOLOGY

Chairman: Dr. R.N. Ginsburg, Fisher Island Station, University of Miami, Miami Beach, FL 33139, U.S.A.

COMMISSION ON THE HISTORY OF GEOLOGICAL SCIENCES (INHIGEO) General Secretary: Dr. E. Dudich, Division of Earth Sciences, UNESCO, Place de Fontenoy, B.P. 3.07, F-75700 Paris, France.

COMMISSION ON IGNEOUS AND METAMORPHIC PETROGENESIS (CIMP) Chairman: Prof. A.J. Naldrett, Department of Geology, University of Toronto, Toronto, Canada, M5S IA I.

COMMISSION FOR MARINE GEOLOGY (CMG)

Seeretary: Prof. J. Thiede, Research Center for Marine Geoseiences at the Christian-Albreehts-University, Wisehhofstrasse 1-3, Building 4, D-2300 Kiel 14, F.R.G.

COMMISSION ON STORAGE, AUTOMATIC PROCESSING AND RETRIEVAL OF GEOLOGICAL DATA (COGEODATA)

Secretary-Treasurer: Dr. G. Gabert, Federal Institute for Geosciences and Natural Resources, P.O. Box 510153, D-3000 Hannover 51, Federal Republic of Germany.

COMMISSION ON STRATIGRAPHY (ICS)

Chairman; Dr. J.W. Cowie, Department of Geology, University of Bristol, Bristol BS8 1TR, U.K.

COMMISSION ON SYSTEMATICS IN PETROLOGY

Chairman: Dr. P.A. Sabine, 19 Beaufort Road, Hanger Hill, Ealing, London W5 3EB, U.K.

COMMISSION ON TECTONICS (COMTEC)

Secretary: Prof. A, Kröner, Johannes Gutenberg-Universität Mainz, Postfach 3980, Saarstrasse 21, 6500-Mainz, F.R.G.

\section{ADVISORY BOARDS}

ADVISORY BOARD FOR RESEARCH DEVELOPMENT

Chairman: Dr. R.W.R. Rutland, Bureau of Mineral Resources, Geology and Geophysics, P.O. Box 378, Canberra, A.C.T. 2601, Australia.

ADVISORY BOARD FOR PUBLICATIONS

Chairman: Dr. J. Aaron, U.S. Geological Survey, Of fice of Seientific Publications, MS904, National Center, Reston VA 22092, U.S.A. ADVISORY BOARD ON REMOTE SENSING

Chairman: Dr. P. Teleki, U.S. Geological Survey, MS917, National Center, Reston, VA 22092 , U.S.A.

\section{COMMITTEES}

ADVISORY COMMITTEE ON THE EVALUATION OF FOSSIL FUELS Chairman: C. Salle, IF P, lA4 Av. de Bois-Preault, 92506 Rueil Malmaison, France.

WORKING GROUP ON PETROLEUM RESOURCE ASSESSM ENT Chairman: Dr. C.D. Masters, U.S. Geological Survey, MS955, National Center, Reston, VA 22092 , U.S.A.

\section{AFFILIATED ORGANIZATIONS}

ASSOCIATION OF ARAB GEOLOGISTS (AGA)

Secretary General: Dr. Sammi Sheriff, Iraq National Oil Company, Al-Khulani Sq., Baghdad, Iraq.

ASSOCIATION OF EUROPEAN GEOLOGICAL SOCIETIES (AEGS) Secretary: Mr. R.M. Bateman, Geological Society of London, Burlington House, Piccadilly, London WIV 0JU, U.K. (To be ratified by IUGS Council). ASSOCIATION OF EXPLORATION GEOCHEMISTS (AEG) Secretary: Dr. Sherman P. Marsh, U.S. Geological Survey, Federal Center, MS-973, Denver, CO 80225 , U.S.A.

ASSOCIATION OF GEOSCIENTISTS FOR INTERNATIONAL DEVELOPMENT (AGID)

jecretary-Treasurer: Dr. Theerapongs Thanasuthipitak, c/o AGID, Asian institute of Technology, Box 2754, Bangkok 10501, 'l'hailand.
ASSOCIATION INTERNATIONALE POUR L'ETUDE DES ARGILES (AIPEA) Secretary General: Dr. A.J. Herbillon, Université Catholique de Louvain, Groupe de Physico-Chimie Minérale et de Catalyse, Place Croix du Sud 1, 1348 Louvain-La-Neuve, Belgium.

ASSOCIATION DES SER VICES GEOLOGIQUES AFRICAINS (ASGA) Technical Secretary: M. Leleu, CIFEG, 103, rue de Lille, 75007 Paris, France.

CARPATHIAN BALKAN GEOLOGICAL ASSOCIATION (CBGA) President: Dr. Z. Dembowski, Centralny Urzad Geologii, Ul. Jasna 6, Warsaw 00-01 3, Poland.

CIRCUM-PACIFIC COUNCIL FOR ENERGY AND MINERAL RESOURCES President and Chairman: Michel T. Halbouty, The Halbouty Center, 5100 Westheimer Road, Houston, Texas 77056, U.S.A.

COMMISSION FOR THE GEOLOGICAL MAP OF THE WORLD (CGMW) Secretary-General: O.Y.J. Dottin, Maison de la Géologie, 77, rue Claude Bernard, 75005 Paris, France.

EUROPEAN ASSOCIATION OF SCIENCE EDITORS (EASE)

Secretary-Treasurer: Ms. Maeve $O^{\prime}$ Connor, 13 Wimpole Street, London W1M $7 \mathrm{AB}$, U.K.

GEOLOGICAL SOCIETY OF AFRICA (GSA)

Secretary-General: F.G. Theuri, Mines and Geological Department, P.O. Box 30009, Nairobi, Kenya.

INTERNATIONAL ASSOCIATION OF ENGINEERING GEOLOGY (IAEG) Secretary General: Dr. M. Primel, L.C.P.C., 58, Bd. Lefebvre, 75732 Paris Cedex 15 France.

INTERNATIONAL ASSOCIATION OF GEOCHEMISTRY AND COSMOCHEMISTR Y (IAGC)

Secretary-General: Dr. B. Hitchon, Alberta Researeh Council, P.O. Box 8330, Station F, Edmonton, Alberta, Canada 'T $6 \mathrm{H} 5 \mathrm{X} 2$.

INTERNATIONAL ASSOCIATION ON THE GENESIS OF ORE DEPOSITS (IAGOD)

Secretary General: Prof. M. Vanecek, Department of Economic Geology, Charles University, Albertov 6, 12000 , Praha 2, Czechoslovakia. Associate Secretary General: Dr. I. Jonasson, e/o Geological Survey of Canada, 601 Booth St., Ottawa, Canada K1A 0E8.

INTER NATIONAL ASSOCIATION OF HYDROGEOLOGISTS (IAH) Secretary General: E. Romijn, Prov. Waterboard of Gelderland, Markstraat 1, P.O. Box 9090, NL-6800 GX Arnhem, The Netherlands.

INTERNATIONAL ASSOCIATION FOR MATHEMATICAL GEOLOGY (IAMG) Secretary General: Dr. R.B. McCammon, U.S. Geological Survey, National Center 920, Reston, VA 22092, U.S.A.

INTERNATIONAL ASSOCIATION OF PLANETOLOGY (IAP)

Secretary: Dr. N. Stovickova, Institute of Applied Geophysics, Podbelohorska 47, 15000 Praha 5, Czechoslovakia.

INTER NATIONAL ASSOCIATION OF SEDIMENTOLOGISTS (IAS)

Secretary-General: Dr. F. Surlyk, Geological Survey of Greenland, Oster

Voldgade 10, Copenhagen $\mathrm{DK}-1350$, Denmark.

INTERNATIONAL FEDERATION OF PALYNOLOGICAL SOCIETIES (IFPS Secretary-Treasurer: Dr. David M. Jarzen, National Museum of Natural Sciences, Paleobiology Division, 1767 Woodward Drive, Ottawa, Canada KlA $0 \mathrm{M} 8$.

INTERNATIONAL MINERALOGICAL ASSOCIATION (IMA)

Secretary-General: Dr. S.S. Hafner, Institute of Mineralogy, University of Marburg, Lahnberge, 3550 Marburg, F.R.G.

INTERNATIONAL PALAEONTOLOGICAL ASSOCIATION (IPA)

Secretary-General: William A. Oliver, Jr., U.S. Geological Survey, E-305 National Museum Building, Washington, DC 20560, U.S.A.

INTERNATIONAL UNION FOR QUATERNARY RESEARCH (INQUA) Secretary-Treasurer: Ch. Schlüchter, Institute of Foundation Engineering, ETH-Hönggerberg, CH-8093 Zurich, Switzerland.

SOCIETY OF ECONOMIC GEOLOGISTS (SEG)

Secretary: Dr. J.F. Murphy, P.O. Box 571, Golden, Colorado 80402, U.S. A.

SOCIETY FOR GEOLOGY APPLIED TO MINER AL DEPOSITS (SGA)

Secretary: Dr. F. Saupé, Centre de Recherches Pétrographiques et

Géochimique, 15, rue N.-D. des Pauvres, 54501 Vandoeuvre, Les Nancy Cedex, France.

INTERNATIONAL GEOLOGICAL CORRELATION PROGRAMME (IGCP) Secretary: Dr. E. Dudich, IGCP, Division of Earth Sciences, UNESCO, Place de Fontenoy, B.P. 3.07, F-75700 Paris, France.

INTER-UNION COMMISSION ON THE LITHOSPHERE (ICL)

Secretary General: Prof. H.L. Zwart, Instituut voor aardwetenschappen, Rijksuniversiteit Utrecht, Budapestlaan 4, Postbus 80.021, 3508 TA Utrecht, The Netherlands.

28th INTERNATIONAL GEOLOGICAL CONGRESS (IGC), 1989 Secretary General: Dr. B. Hanshaw, International Geological Congress, P.U. Box 1001, Herndon, VA 22070, U.S.A. 